NASA Technical Memorandum 100133

ICOMP-87-4

\title{
Vortex-Scalar Element Calculations of a Diffusion Flame Stabilized on a Plane Mixing Layer
}

\author{
Ahmed F. Ghoniem \\ Massachusetts Institute of Technology \\ Cambridge, Massachusetts \\ and \\ Peyman Gini \\ Institute for Computational Mechanics in Propulsion \\ Lewis Research Center \\ Cleveland, Ohio
}

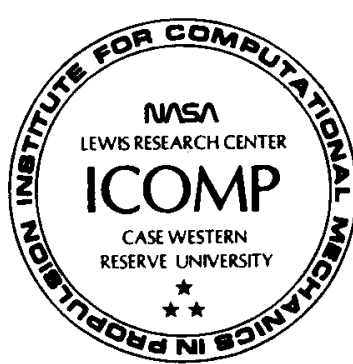

CN ALANE HIXING LAYER (NASA) $26 \mathrm{p}$

Avail: $\triangle T I S \mathrm{HC}$ AC3/MF AO1 CSCL 01A

N87-28501 
VORTEX-SCALAR ELEMENT CALCULATIONS OF A DIFFUSION FLAME STABILIZED ON A

\title{
PLANE MIXING LAYER
}

\author{
Ahmed F. Ghoniem* \\ Department of Mechanical Engineering \\ Massachusetts Institute of Technology \\ Cambridge, Massachusetts 02139
}

and

Peyman Givi**

Institute for Computational Mechanics in Propulsion

Lewis Research Center

Cleveland, Ohio 44135

\begin{abstract}
SUMMARY
The vortex-scalar element method, a scheme which utilizes vortex elements to discretize the region of high vorticity and scalar elements to represent species or temperature fields, is utilized in the numerical simulations of a two-dimensional reacting mixing layer. Computations are performed for a diffusion flame at high Reynolds and Peclet numbers without resorting to turbulence models. In the nonreacting flow, the mean and fluctuation profiles of a conserved scalar show good agreement with experimental measurements. Results for the reacting flow indicate that for temperature-independent kinetics, the chemical reaction begins immediately downstream of the splitter plate where mixing starts. Results for the reacting flow with Arrhenius kinetics show an ignition delay, which depends on the reactants temperature, before significant chemical reaction occurs. Harmonic forcing changes the structure of the layer, and concomitantly the rates of mixing and reaction, in accordance with experimental results. Strong stretch within the braids in the nonequilibrium kinetics case causes local flame quenching due to the temperature drop associated with the large convective fluxes.
\end{abstract}

\section{INTRODUCTION}

Turbulent diffusion flames have been the subject of extensive experimental and theoretical investigations during recent years (for a review, see Bilger (ref. 1)). In most of the theoretical work, turbulence models are used to close a system of averaged transport equations which describes the statistical behavior of the aerothermodynamical variables. Moment methods (ref. 2), eddy break-up and mixing controlled models (ref. 3), flame sheet approximation (ref. 4), assumed probability density function (PDF) shape methods (ref. 5), solutions based on modeled joint PDF of scalar quantities (refs. 6 and 7 ), and based on modeled joint PDF of scalar and velocity (ref. 8) are examples in

*Work supported by the Air Force Office of Scientific Research Grant AFOSR 840356 , the National Science Foundation Grant CPE-840481, and the Edgerton Associate Professorship at Massachusetts Institute of Technology.

**Work funded under Space Act Agreement C99066G; on leave from Flow Research Company, Kent, Washington. 
which turbulence modeling have been used for the closure of equations governing the statistical quantities. Much effort has gone into constructing accurate models and in obtaining results that are in agreement with experimental measurements. However, in complex systems, modeling is difficult because of our lack of knowledge on the detalled dynamics of the flow. Furthermore, since most of the interesting dynamical behavior of the flow is modeled a priori, such features are not exhibited from the results of numerical computations based on turbulence models, and thus can not advance our understanding of turbulent combustion.

The progress in numerical methods and the availability of supercomputers have had a major impact on turbulence research. Improved accuracy of the numerics and increased storage and computational speed have made it possible to solve the appropriate transport equations governing turbulent combustion indirectly without the need for modeling over some limited parameter range. Such nearly model-free "simulations," in comparison with calculations utilizing turbulence models, have the advantage that the dominant physics of the problem is not modeled a priori, but is recovered directly from the computed results. Their results can be used to understand many important mechanisms of turbulent transport and its direct influence on chemical reactions. Furthermore, since the instantaneous behavior of the variables are known at all points and at all times, accurate simulations offer a good method of probing the flow when experimental techniques may fail. There are, however, some limitations on the range of turbulent scales that can be resolved accurately by model free simulations. Therefore, there is a need to validate the results of the simulations by a direct comparison with experimental measurements. With such validations, ab initio predictions can ultimately be a reality.

Numerical methods have been used in a variety of forms for the simulation of turbulent flows in complex configurations. A recent survey can be found in review articles (refs. 9 and 10). In reacting flow, three approaches are used: (1) finite difference methods, (2) spectral methods; and, (3) vortex methods. In the first approach, the variables are defined on a grid and the transport equations are approximated by discretizing the derivatives on the grid nodes. Examples of this approach can be found in the work of corcos and Sherman (ref. 11) who used a projection method to study the temporal evolution of a periodic shear layer, and in Grinstein et al. (ref. 12) who used a flux-corrected transport scheme to simulate the development of coherent structures in a two-dimensional spatially evolving shear layer and examined their effect on mixing.

In spectral methods, the variables are expanded in series of harmonic functions that satisfy the differential equations on a number of collocation points. Riley et al. (ref. 13) used a pseudo-spectral scheme to study a threedimensional temporaliy-evolving reacting mixing layer assuming a constant reaction rate, constant density, and no heat release. McMurtry et al. (ref. 14) considered the effects of the chemical heat release on the fluid dynamics of a two-dimensional mixing layer for a constant reaction rate. The interplay between fluid dynamics and the chemical reaction is investigated under these conditions. Givi et al. (ref. 15) used the same method to compute a twodimensional mixing layer with an Arrhenius chemical reaction and constant density to assess the effects of large coherent structure on the local extinction of the flame. Extension to spatilly-growing layers was initiated by Givi and Jou (ref. 16) using a hybrid pseudo-spectral second order finite difference scheme. In all cases, the Reynolds number was kept at small values, $0(100)$, limited by the grid resolution and the number of harmonic modes. 
In the third approach, vortex methods are used. These schemes are grid free, the transport of the variables takes place in a Lagrangian form, and the solution is not restricted by the geometry of the confinement. Therefore they can provide accurate simulations for high Reynolds number, spatially growing flows. Moreover, vortex methods optimize the computational efforts by distributing computational elements around regions of high vorticity. The application of the method in thin premixed flame calculations with a finite density jump has been reported by Ghoniem et al. (ref. 17) and Sethian (ref. 18), among others. In these calculations, the vortex method was employed to compute the flow field, and the dynamic effect of combustion was represented by the propagation of a thin interface at the laminar burning velocity acting as a volumetric source.

Vortex methods were also used in simulating diffusion flames in connection with a finite-difference approach for the treatment of the scalar variables. Ashurst and Barr (ref. 19) used the vortex method to compute the hydrodynamic field and an Eulerian flux-corrected transport algorithm to compute the diffusion and convection of a conserved Shvab-Zeldovich scalar approximating the shape and convolution of the flame in the limit of infinitely fast chemical reaction. Lin and Pratt (ref. 20) used the random vortex method to simulate the large-scale motion and a Monte-Carlo method to calculate the time-dependent probability density function of the scalar quantities for both gaseous and aqueous mixing layers. The PDF transport equation, however, required a closure model for the molecular mixing term.

From this short review, it is clear that numerical simulations have played an important role in elucidating the physics of turbulent reacting flows, and that there is a continuing need for more direct simulations in order to explain better some of the interesting physical phenomena that have been observed in laboratory experiments.

In this work, we extend the vortex method to study nonpremixed chemical reactions. A vortex-scalar element method is developed to treat both the hydrodynamic and the scalar field in a Lagrangian sense. The fact that a chemical reaction is truly a Lagrangian process, $1 . e .$, it occurs when the particles (or macroscopic elements) interact as they flow, motivate the implementation of Lagrangian methods for simulations of high Reynolds number reacting flows. The method is capable of handing a wide variety of initial and boundary conditions and is not limited to simple flow boundaries. In this paper, we concentrate on the formulation of the model and the numerical schemes, and present some preliminary validation studies and interpretations of the results.

In section 2, the geometrical configuration of a spatially evolving mixing layer is presented, and the formulation of the problem and of the scheme are described. Results of some sample calculations are given in section 3 . Computations of a nonreacting mixing layer is performed first in order to check on the accuracy of the method by comparing its results with experimental measurements at the same conditions. Preliminary results of a reacting mixing layer simulation in which the two reactants are introduced in different streams are presented next. Both constant rate kinetics and temperature dependent kinetics are considered. In both cases, the influence of the coherent structures on the finite rate chemistry is assessed and in the second case, the nonequilibrium effects in the reaction rate are examined. In the constant rate kinetics calculations, the influence of harmonic forcing at the inlet of the mixing layer is investigated. This study was motivated by recent experimental observations 
of Roberts and Roshko (ref. 22) and numerical computations of Ghoniem and $\mathrm{Ng}$ (ref. 23). The paper is concluded in Section IV with a summary of our new results and suggestions for future developments.

\section{FORMULATION AND NUMERICAL SCHEME}

A two-dimensional, confined, planar mixing layer is considered. A schematic diagram of the flow field is shown in figure 1. Two initially unmixed reactants, fue $1 \mathrm{~F}$ and oxidant 0 , are present at small concentrations in the top high speed stream and bottom low speed stream, respectively. We make the fol-. lowing assumptions: (1) the heat release is low so that its effect on the dynamics of the flow is negligible; (2) the Mach number is small; (3) the free stream concentrations of $F$ and 0 are equal and constant; (4) the molecular diffusivities are equal and constant; (5) the viscosity is the same in both streams; and (6) the chemical reaction between $F$ and 0 is single step, irreversible, and second order. The density is, therefore, constant, and the transport equations of the hydrodynamic field and the scalar - temperature or species -- fields are decoupled. The equations governing this system are:

$$
\begin{aligned}
& F+ \stackrel{k}{\rightarrow} p \\
& \nabla^{2} \Psi=-\omega(x, t) \\
& \frac{\partial \omega}{\partial t}+u \cdot \nabla \omega=\frac{1}{R e} \nabla^{2} \omega \\
& \frac{\partial T}{\partial t}+u \cdot \nabla T=\frac{1}{P e} \nabla^{2} T+Q D a \dot{W} \\
& \frac{\partial c_{j}}{\partial t}+u \cdot \nabla c_{j}=\frac{1}{P e ~ L e} \nabla^{2} c_{j}-D a \dot{W} \\
& \frac{\partial c_{p}}{\partial t}+u \cdot \nabla c_{p}=\frac{1}{P e ~ L e} \nabla^{2} c_{p}+D a \dot{W}
\end{aligned}
$$

where $P$ indicates products and $\dot{W}=C_{F} C_{0} \exp (-T a / T)$ is the reaction rate, written in terms of the rate of generation of products per unit mass. $u=(u, v)$ is the velocity, $x=(x, y)$ and $x, y$ are the streamwise and cross stream directions, respectively, $t$ is time, $\psi$ is the stream function defined such that $u=\partial \psi / \partial y$ and $v=-\partial \psi / \partial x, \omega=\nabla \times u$ is the vorticity, $c$ is the concentration per unit mass, $T$ is temperature. $\nabla=(\partial / \partial x, \partial / \partial y)$, and $\nabla^{2}=\partial^{2} / \partial x^{2}+\partial^{2} / \partial y^{2}$. Variables are nondimensionalized with respect to the appropriate combination of the total shear $\Delta U=U 1$ - U2, the channel height $H$, the free stream concentration of $F, C_{F o}$, the free stream temperature at $x=0$, To. In equation (5), $J=F$ or 0 for fuel and oxidizer, respectively. $\operatorname{Re}=\Delta U \mathrm{H} / v$ is the Reynolds number, where $v$ is the kinematic viscosity. The reaction rate constant $k=A \exp (-\mathrm{Ta} / T)$ where $A$ is the frequency factor, and $\mathrm{Ta}$ is the activation energy, nondimensionalized with respect to (RTO), $R$ being the gas constant. $Q$ is the enthalpy of reaction, nondimensionalized with respect to $C_{p T o}$, where $C_{p}$ is the specific heat at constant pressure. $\mathrm{Pe}=\Delta U \mathrm{H} / \alpha$ is the Peclet number, where $\alpha$ is the thermal diffusivity. 
$\mathrm{Da}=\mathrm{A} \mathrm{C}_{\mathrm{FO}} \mathrm{H} / \Delta \mathrm{U}$ is the first Damkohter number. $D$ is diffusivity and Le $=\alpha / D$ is the Lewis number.

Since equations (4) to (6) are similar, there is no need to solve them all if the scalar concentrations $c_{F}, c_{0}$, and $c_{P}$ are normalized in such a way that their initial and boundary conditions are identical. This is accomplished by the use of Shvab-Zeldovich transformation (ref. 1). Introducing conserved scalars $B_{F P}=C_{F}+c_{P}$, and $B_{O P}=1-\left(c_{0}-c_{P}\right)$, we get:

$$
\frac{\partial \beta_{j}}{\partial t}+u \cdot \nabla \beta_{j}=\frac{1}{P e \text { Le }} \nabla^{2} \beta_{j}
$$

for $j=F P$ or $O P$. Since $B_{F P}$ and $B_{O P}$ have the same initial and boundary conditions, $B_{F P}=B_{O P}=B$. The finite rate kinetics effects can be taken into account by considering the transport equation for the product of chemical reaction, equation (6), and equation (7) for a conserved scalar. If the Lewis number is unity, another conserved scalar can be introduced, $B_{P T}=c_{P}-T / Q$, and the solution of equations (6) and (7) for $c_{p}$ and $B$ will determine the behavfor of all the scalar equantities, $C_{F}, C_{0}, C_{P}$, and $T$.

\subsection{The Vortex Scheme}

In the vortex method, the vorticity field is represented by a finite number of vortex elements of finite cores:

$$
\omega(x, t)=\Sigma \Gamma_{j} / \delta^{2} f\left(x-x_{j}\right)
$$

where $r_{i}=\int \omega d A$, is the circulation of a vortex element and $\delta$ is the core radius, while $x_{j}$ is the center of the element. $f$ represents the voriticity distribution associated with a vortex element, or the core function (Chorin (ref. 23), Hald (ref. 24), and Beale and Majda (ref. 25).) The velocity field is obtained by solving equation (2) using the discrete vorticity distribution.

$$
u=\Sigma \Gamma_{i} K\left(x-x_{j}\right) k\left(x-x_{j}\right)+u_{p}
$$

where $K(x)=-(y,-x) / r^{2}$ is the kernal of the Poisson equation, $x(x)=\int r f(r) d r$ is the circulation within $r$, and $r=|x|$. up is an irrotational velocity fleld added to satisfy the potential boundary condition; $u_{p}=\nabla_{\phi}$ where $\nabla^{2} \phi=0$ and $u \cdot n=0$ on solid boundaries while $u . n=U$ at the inlet, $n$ is the normal unit vector. For the confined shear layer, the boundary condition at $x=0$ is: $u=U 1$ for $y>0$ and $u=U 2$ at $y<0$, while $y=0$ is a vortex sheet of strength $\Delta U=U 1-U 2$.

In this work, we use Rankine vortex elements, i.e., the vorticity of an element is constant within the core and zero outside, $f(r)=1 / \pi$ for $r \leq \delta$ and $f(r)=0$ for $r>\delta$. Correspondingly, $k(r)=r^{2} / 2 \pi$ for $r \leq \delta$ and $\kappa=1$ for $r>\delta$. Moreover, the potential velocity field is obtained by conformal transformation. Thus, the physical plane is mapped onto the upper half plane and image vortices are used to satisfy the potential boundary conditions. The form of the mapping function for the confined shear layer is given by Ghoniem and $\mathrm{Ng}$ (ref. 22). 
The motion of the vortex elements must be constructed such that the vorticity field satisfies equation (3). This is accomplished by solving this equation in two fractional steps:

$$
\begin{aligned}
& \text { Convection: } \frac{\partial \omega}{\partial t}+u \cdot \nabla \omega=0 \\
& \text { Diffusion: } \frac{\partial \omega}{\partial t}=\frac{1}{\operatorname{Re}} \nabla^{2} \omega
\end{aligned}
$$

In the first step, the convective transport of vorticity is implemented in terms of the Lagrangian displacement of the vortex elements using the current velocity field computed from equation (9). In the second step, the solution of the diffusion equation is simulated stochastically by the random walk displacement of the vortex elements according to the appropriate population. Thus:

$$
x_{i}(t+\Delta t)=x_{i}(t)+\varepsilon_{k} u\left(x_{i k}\right) \Delta t=\eta_{i}
$$

for $1=1,2, \ldots, N$, where $\Sigma_{k}$ is a $k$-th order time-integration scheme and $n 1$ is a two-dimensional Gaussian random variable with zero mean and standard variation $\sqrt{2 \Delta t / R e}$. For more details, see Ghoniem and $\mathrm{Ng}$ (ref. 22), Ghoniem and Gagnon (ref. 26).

The no-silp boundary condition at the walls is satisfied by generating new vortex elements to cancel the induced velocity by the vorticity field. Here, we generate vorticity only at the point of separation, i.e., at the tip of the splitter. plate since the growth of the boundary layers along the channel walls at these high Reynolds numbers is small. At each time step, the new vorticity $\Delta \Gamma=-\Delta U U m \Delta t$, where $U m=(U 1+U 2) / 2$, is consigned to No elements of strength $\Delta \Gamma /$ No and added to the field at points $\Delta x=$ Um/No apart downstream of $x=0$.

The effect of the numerical parameters on the accuracy of the results was investigated by Ghoniem and $\mathrm{Ng}$ (ref. 22). Their results emphasized the importance of using a high order time-integration scheme with $k=2$ to avoid excessive numerical diffusion in the vorticity field. The value of No $=6$ was also found to be appropriate in order to obtain well-defined eddy structures after the rollup and the first two pairings. The second pairing is accomplished within the domain of $0 \leq x \leq 6$, therefore the computational domain was limited to $x_{\max }=6$. Downstream of $x_{\max }$, the vorticity was deleted. varying $x_{\max }$ showed that the effect of deleting the vortex elements propagates about one channel highest upstream, hence the results are accurate only for $0 \leq x \leq 5$.

\subsection{THE SCALAR FLEMENT METHOO}

In this scheme, which is a two-dimensional extension of the random element method of Ghoniem and Oppenheim (ref. 27), the scalar field is represented by a set of elements each carrying a finite amount of the scalar field.

$$
s(x, t)=\Sigma s_{j} \delta\left(x-x_{j}\right)
$$


where $s$ is a scalar field, being the temperature of species concentration, $s_{1}$ is the strength of an element, defined as the amount of scalar carried by this element and $\delta($.$) is the Dirac delta function, s_{j}=1 / \delta A s s(x, t) d A$, where $\delta A=\delta x \delta y$, and $\delta x$ and $\delta y$ are the distances between the centers of neighboring elements in the streamwise and cross stream directions, respectively, and $x_{i}$ is the center of the element. If $s$ is the active scalar, its transport is governed by:

$$
\frac{\partial s}{\partial t}+u \cdot \nabla s=\frac{1}{S e} \nabla^{2} s+\dot{w}
$$

where Se is the ratio between the diffusive and convective time scales of transport of $s$, Se $=P e$ for $s=T$, and $S e=P e$ Le if $s=c$. In the scalar element method, this equation is solved in three fractional steps:

$$
\begin{aligned}
& \text { Convection: } \frac{\partial S}{\partial t}+u \cdot \nabla s=0 \\
& \text { Oiffusion: } \frac{\partial S}{\partial t}=\frac{1}{S e} \nabla^{2} s \\
& \text { Reaction: } \frac{\partial S}{\partial t}=\dot{W}
\end{aligned}
$$

Convective and diffusive transport are taken into account in a similar way as in the vortex method, 1.e., by the Lagrangian motion of the scalar elements using the velocity field $u$, computed using equation (9), and the random walk displacement of the elements using a set of Gaussian random variables with zero mean and standard deviation $\sqrt{2 \Delta t / S e}$ (Ghoniem and Sherman (ref. 28)). If $x_{i}$ is the center of the element $i$, then,

$$
x_{j}(t+\Delta t)=x_{j}(t)+\Sigma u\left(x_{j k}\right) \Delta t+n_{j}
$$

Chemical reaction changes the amount of reactants carried by the element according to the integration of equation (17).

$$
s_{i}(t+\Delta t)=s_{i}(t)+\dot{W} \Delta t
$$

However, the reaction occurs only when the element are close enough for molecular mixing to affect their composition. Therefore, at every time step, the distance between the centers of each two elements of $F$ and 0 $\Delta x_{i j}=\left|x_{i}-x_{j}\right|$ is computed. If $\Delta x_{i j} \leq \delta_{D}$, where $\delta_{D}=0\left(1 \sqrt{S_{e}}\right)$ is the diffusion length scale, the composition of each of the two element changes according to equation (19). The initial distance between neighboring elements must be small enough to allow enough interactions between the elements. This limits the maximum value of the Peclet number that can be economically used in the computations to $0(1000)$.

The scheme, while providing an approximate solution of equation (12) in a stochastic sense, mimics closely the actual physics of the reaction process. This is achieved by using the Lagrangian formulation of the transport equations and dealing with the chemical production terms in individual particles. 


\section{RESULTS AND DISCUSSION}

The computer code, developed by Ghoniem and $\mathrm{Ng}$ (ref. 22) for vortex simulation of a nonreacting shear layer, was vectorized in order to take advantage of the computational capability of a CRAY-XMP. The scheme, being explicit in time and requiring mostly nonrecursive computations, can utilize this capability efficiently. The dynamics for the nonreacting layer was investigated in detail in the work of Ghoniem and $\mathrm{Ng}$ (ref. 22). Here we concentrate on results pertaining to mixing and to chemicaliy-reacting layer.

\subsection{Nonreacting Mixing Layer}

Results of a typical simulation, presented in terms of the velocity and location of all vortex elements used in the computations, are shown in figures 2 to 4 for the cases of $R e=24000, \operatorname{Re}=4000$, and $\operatorname{Re}=1000$, respectively. Each vortex element is depicted by a point, while its velocity relative to the mean velocity is represented by a line vector starting at the center of the vortex element. The velocity ratio across the layer at the inlet is $\mathrm{U} 2 / \mathrm{U} 1=1 / 3$.

Results show the formation of large vortex eddies by the rollup of the vorticity layer that emanates at the splitter plate, and the subsequent pairings of these eddies into larger structures. The rollup of the shear layer was investigated in Ghoniem and $\mathrm{Ng}$ (ref. 22) by analyzing results at a wide range of the Reynolds number and at different boundary conditions. Their analysis show that: (1) the rollup is due to the growth of perturbations by the KelvinHelmholtz instablitty mechanism, and the shedding frequency corresponds to the most unstable frequency predicted from the linear stability analys is of a spatially growing layer; (2) pairing, which is associated with the local subharmonic perturbations, results in a step-wise increase in the size of the vorticity layer as two eddies merge; (3) The two sources of the subharmonic perturbations are the downward motion of the layer and the monotonic growth in the size of the eddies downstream; (4) the intrinsic dynamics of the instability is not strongly affected by the value of the Reynolds number, except that at the low Reynolds number the eddies are slightly larger due to the disperison of vorticity by diffusion; and (5) the computed velocity statistics show good agreements with experimental data, indicating that the fundamental mechanisms of the shear layer are two-dimensional and, hence, the numerical scheme is capable of predicting the large scale features accurately.

The study entrainment, a passive conserved scalar with a normalized concentration value equal to zero in the high speed stream and equal to one in the low speed side is introduced at the inlet section. At each time step, 19 elements are introduced in each stream. The initial distance between two neighboring elements in the cross stream direction is taken as $\delta y=0.021$. The time step $\Delta t=0.1$, thus the distance between the elements in the streamwise direction is $\delta x=0.05$ on the average. Since diffusion is more critical in the cross stream direction, $\delta y$ is chosen to be smaller than $\delta x$. A case with $\delta y=0.016$, using 25 elements in each stream was computed, showing no significant change in the overall behavior.

Figures 5 to 7 are obtained for Reynolds number, Peclet number, and velocity ratio 10000,4000 , and 1/2, respectively. Figures 5 and 6 show the velocity and location of all the vortex and scalar elements respectively, while 
figure 7 exhibits the strength of each of the scalar elements at the nondimensional times of $t=28,29$ and 30 . In figure 6 , the dots represent the fluid from the high speed side with normalized concentration $c=0$, and the open circles represent the fluid from the low speed side with $c=1$. This figure indicates that the rollup of the vortices and their subsequent pairing entrains fluid from both sides of the free streams into the cores of the vorticity layer, which results in the enhancement of mixing between the two streams. Entrainment asymmetry is observed as more fluid from the high speed side is present in the low speed side than the opposite (Koochesfahani (ref. 29)).

The instantaneous profiles of the concentration field are averaged over a long-time period and the statistical values are compared with experimental data in figures 8 and 9 . Figure 8 shows the mean value of the concentration, $\bar{c}_{m}$, as a function of $\left(y-y_{0}\right) /\left(x-x_{0}\right)$, where $y_{0}$ is measured at $\bar{c}_{m}=0.5$ and $x_{0}$ is the virtual origin of the mixing layer based on the mean concentration profile (in the calculation, $x_{0}=0$ ). In this figure, the solid line is the computed mean concentration at $x=4$ and the data points are obtained from recent experimental measurement by Masutani and Bowman (ref. 30) for a dilute nonreacting mixing layer with the same velocity ratio. Figure 9 shows a comparison between the computed and measured mean fluctuations of the concentra-

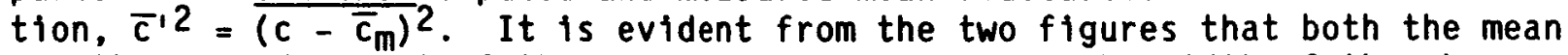
and the second moment of the conserved scalar across the width of the shear layer are accurately predicted by our computations.

We note that the results in figures 8 and 9 are in better agreement with experimental data than those previously predicted by Givi et al. (ref. 31). In these calculations, a $k-\varepsilon$ turbulence model and a gradient diffusion mode 1 for turbulent transport of the scalar mean, moment, and probability density function was utilized. In the $k-\varepsilon$ calculations, the concentration fluctuations exhibit a fairly smooth bell-shaped profile with a much less clear double "hump" in the middle region, indicating poor agreement near the high speed stress. The present calculations show the two local maxima in the fluctuation profiles that correspond to the location where the gradient of the mean value is highest. The same behavior is observed by the experimental results of Masutani and Bowman (ref. 30) and Batt (ref. 32). It is clear that, in accordance with the findings of Broadwell and Briedenthal (ref. 33), the intermittency caused by the large coherent structures contributes greatly to the statistics of this turbulent flow.

\subsection{Reacting Mixing Layer}

In the calculation of a reacting mixing layer, two reactants $F$ and 0 are introduced on both sides of the splitter plate. At $x=0$, for $y>0$, $c_{F}=1$, and $c_{0}=0$, and for $y>0, c_{0}=1$, and $c_{F}=0$, while $c_{P}=0$. As reactants are entrained into the mixing cores of the layer, they diffuse across the original interface and chemical reaction proceeds. The rollup and pairing increases the original length of the interface by many folds and allow the entrained fluid to diffuse along a larger boundary (Ghoniem et al. (ref. 34)). During this process, if the Lagrangian elements utilized to represent the interaction between chemically reacting species are brought close enough so that the distance between two neighboring elements is smaller than the characteristic diffusion length, they react at the rate defined by equation (17). 
In figures 10 to 12 , we present the velocity, location, and the strength of the elements in terms of product concentration for the reacting mixing layer with constant rate chemical kinetics and temperature-dependent reaction rate, respectively. The amount of the products formed due to chemical reaction is presented by the diameter of the circles in the figures, 1.e., larger circles indicate more products. In both cases, $\mathrm{Re}=10000, \mathrm{Pe}=4000$, and $\mathrm{U} 2 / \mathrm{UT}=1 / 3$ while $\mathrm{Le}=1$. In the constant rate $\mathrm{kinetics}$ case, the value of the Damkohler number $\mathrm{Da}=1$ and in the temperature-dependent $k$ inetics $\mathrm{Da}=$ 200, $\mathrm{Ta}=10$, and $Q=5$. Note that in both cases the value of the nondimensional kinetic parameters are low enough so that the effects of heat release on the fluid dynamics can be negligible. The stiffness of equation (19) for large values of the Damkohler number imposes a restriction on the time step of integration. In these calculations, we found that $\Delta t=0.1$ is sufficientiy small to accurately integrate the slow chemistry.

A comparison between the two figures reveal that under isothermal cond $i$ tions, the products are formed as mixing occurs just downstream of the splitter plate, while in the temperature-dependent kinetics calculations, there is an ignition delay before the reactant reach a temperature high enough to allow any significant chemical reaction to occur. Once the reaction begins, the mechanism of product formation and chemical reaction in both cases are asymptotically the same. Increasing the Damkohler number to $\mathrm{Da}=400$ results in a shorter ignition delay, and preheating the reactants by increasing the temperature at the inlet to $\mathrm{TI}=\mathrm{Q} / 2$ while $\mathrm{Da}=200$, eliminates the ignition delay as ind $i$ cated in figures 13 and 14 , respectively.

In order to examine the effects of chemical reaction on the transport of species, the concentration statistics in the temperature-independent reaction case are presented in figures 15 and 16 . These figures correspond to the ensemble mean and fluctuations in the bottom-stress species concentration in a reacting mixing layer with $\mathrm{Da}=1, \mathrm{U} 2 / \mathrm{U} 1=1 / 2, \mathrm{Re}=10000$, and $\mathrm{Pe}=4000$. A comparison between figures 15 and 8 , and between figures 16 and 9 indicates that near the free stream, the chemistry does not affect the statistical behavfor of the species. Near the reaction zone, however, the mean and the rms values of the concentration are lower under reacting conditions, while the second hump near the high speed stream side of the rms profile in the nonreact ing layer is eliminated in the reacting flow due to the local consumption of the species by chemical reaction. The same behavior was also observed in the experiments of Masutani and Bowman (ref. 30) in a reacting mixing layer under isothermal conditions. Their results, however, can not be compared quantitatively with the present calculations since the values of the chemical parameters employed in the numerical simulation are substantially lower than those of the experiment.

\subsection{Effect of Harmonic Forcing}

The dynamic effect of oscillating the upstream side of the layer was studied experimentally by several authors, e.g., Oster and Wygnanski (ref. 35) and Roberts and Roshko (ref. 21) and numerically by Ghoniem and Ng (ref. 22). Their results indicate that in the forced case, eddy interactions follow four stages. In the first stage, the layer rolls up at the harmonic of the forcing frequency closest to the most amplified mode. In the second stage, a process of accelerated pairings yields a large eddy which is in tune with the forcing frequency. This large resonant eddy appears earlier than it would appear in 
the case of an unforced layer. In the third stage, paring among resonant eddies, which represents a neutrally stable mode, is disabled and the growth of the vorticity layer is impaired for several eddies downstream. In the fourth stage, the effect of forcing diminishes and pseudo-random pairing is resumed. Moreover, velocity statistics are affected by forcing, and the sign of momentum transfer across the layer is reversed following pairing. Entrainment of passive particles was found to be commensurate with the development of the vorticity layer.

In the recent experiment by Roberts and Roshko (ref. 21 ), it has been observed that periodic forcing has a direct influence on the outcome of chemical reaction across a turbulent shear layer. The results of this experiment indicate that when harmonic forcing is applied, the mixing rate: (1) is increased in the initial stages where the resonant eddy is forming; (2) is decreased in the intermediate stage which corresponds to the resonant or "frequency-locked" region; and, (3) is the same as that of the unforced layer further downstream. In order to characterize these three regions, the Wygnanski-0ster parameter $X_{W}=\Delta U \Omega x / U m^{2}$ is utilized, where $\Omega$ is the forcing frequency (ref. 35). Roberts and Roshko (ref. 21) and Browand and Ho (ref. 36) show that the three different regions can be classified according to the local value of $X_{W}$ parameter. In region $I, X_{W}<1$, the growth rate is enhanced.

In region II, $X_{W}>1$, the frequency-locked region, the growth rate is inhtbited. In region III, the growth rate relaxes to that of the unforced layer.

In order to investigated this phnomenon computationally, the response of the reacting shear layer to the application of low frequency, low amplitude perturbations on the upstream side of the shear layer is computed. Streamwise oscillations are applied on both sides of the layer, hence a pressure preturbation is imposed without changing the vorticity field. The streamwise velocities are taken as $U 1=1+a \sin (2 \pi \Omega t)$, and $U 2=\alpha U 2$, where a is the amplitude of forcing.

The normalized distribution of the product thickness along the mixing layer for three cases, $\Omega=0,0.5$, and 1 , is shown in figure 17 . In these calculations, $a=0.1$, and $R E=4000$. The figure indicates that for $\Omega=1$, mixing is enhanced in the initial part of the layer, $1 \leq x \leq 2$. The resonant, frequency-locked region begins at $x=2$ and ends at value $x \sim 3$. In this region, mixing is reduced and is less than that of unforced mixing layer. Downstream of this region, $x \geq 3$, mixing rate resumes its natural growth and reaches asymptotically that of the unforced layer. For lower forcing frequency, $\Omega=0.5$, the same overall behavior is observed. In this case, however, the results of numerical calculations indicate that the resonant frequency-locked region is approximately in the range $3 \leqq 4 \times \leqq 4$. A comparison between the range of the frequency locked region calculated here with that estimated by Browand and Ho (ref. 36) is shown on table I. Considering the fact that our simulations ignore the effect of small scale three-dimensional turbulence motion, and considering the nonuniversality of the Browand and Ho's curve due to its independence to experimental conditions and other important nondimensionalized parameters, this agreement is encouraging. 


\subsection{Effects of Strain Rate}

It has been shown experimentally by Tsujt (ref. 37 ), numerically by Liew et al. (ref. 38), and analytically by Peters (ref. 39), that the strain rate has a major influence on the flame structure, particularly in nonpremixed systems. In the counter-flow diffusion flame experiments of Tsuji (ref. 37), it was observed that increasing the magnitude of stretch near the flame surface results in an increase of the flow of reactants into the reaction zone. As a result, the chemical reaction is not able to keep pace with the supply of reactants, and the reaction rate is reduced unt 11 local flame quenching occurs. The analysis of Peters (ref. 39), which is based on the method of matched asymptotic expansion at large activation energy, shows that the mechanism of flame extinction can be addressed by examining the local value of the rate of scalar dissipation. This parameter is viewed by Peters (ref. 39) as the inverse of the diffusion time scale. If the local value of dissipation is increased beyond a critical limit, the heat conducted away from the diffusion flame can not be balanced by the heat produced by the chemical reaction. As a result, the maximum value of the temperature decreases, and the reaction eventually ceases.

By increasing the number of scalar elements to 38 in each stream while decreasing the computational domain to $X_{\max }=4$, and by preheating the incoming reactants to $\mathrm{TH}=\mathrm{Q} / 2$ to start the chemical reaction immediately down. stream the splitter plate, we were able to observe this phenomenon. Figures 18 and 19 show the instantaneous velocity and temperature rise, T - Ti, of the scalar element at times of $t=19$ and $t=21$, respectively. In this case, the Damkohler number, the normalized enthalpy of reaction, the activation energy, and the velocity ratio at the inlet are $50,8,20$, and $1 / 3$, respectively. The cross-stream direction is enlarged by a factor of 2 for the purpose of clarity.

The figures show that the number of scalar elements near the braid, which is the thin link between two neighboring cores, is only a small portion of the total number of elements within the computational domain, which reached more than 5100. This indicates an instantaneous quenching at the stagnation points of the layer. Moreover, the temperature and product concentration in the reaction zone reach a maximum at the core of the eddies where the vorticity concentration is high, while they reach a minimum at the stagnation point within the braid between the neighboring cores where the strain and the scalar gradients reach their maximum values. This is consistent with the results of the pseudospectral calculations of Givi et al. (ref. 15), and with the experimental observations of Tsuji (ref. 37) who showed that the local extinction of diffusion flame occurs mainly at the regions of high dissipation rate. At these regions, the temperature tends to decrease, and if it goes below a critical characteristic value, the flame localiy extinguishes.

Quantitative analysis of the effects of stretch on the chemical reaction is rather difficult in the context of present algorithm. This is due to the fact that there are very few scalar elements near the regions of high strain, and as shown by Ghoniem et al. (ref. 34), most on the elements tend to be concentrated near the regions with low dissipation. Implementation of a numerical scheme based on the transport of the scalar gradients, as in Ghoniem et al. (ref. 34) can improve the accuracy of the analysis substantially, particularly those associated with the effects of stretch. In this method, the elements are concentrated near the regions of large gradients, or high dissipation, and 
hence a smaller total number of elements have to be considered. The implementation of this method for the numerical simulation of unpremixed reacting flows is presently underway to study the effect of strain rate more accurately.

\section{CONCLUSION}

In this work, a numerical scheme based on the transport of computational element carrying vorticity and scalar quantities has been developed to simulate a reacting planar, two-stream mixing layer with unmixed reactants. The scheme solves the transport equations at high Reynolds and Peclet numbers without using models for turbulence closure. A Lagrangian stochastic model is used to implement the chemical reactions for both constant rate kinetics and variable temperature Arrhenius reactions.

In the nonreacting flow simulations, the calculated statistics of the mixing of a conserved scalar are in good agreement with experimental data. In particular, the numerical results show the presence of two maxima in the fluctuation profile. In the constant rate reacting flow simulation, the effect of chemistry is to smooth out this curve and produce a single maximum, which agrees with the experimental observations. Harmonic forcing enhances the mixing within the accelerated growth zone of the vorticity layer, while it impairs the entrainment of the unmixed fluid into the cores in the resonating region. As a result, the numerical simulation indicates a decrease in the rate of produce formation in the frequency-looked region, similar to previous experimental findings.

In the Arrhenius, temperture-dependent kinetics, the mechanism of ignition delay, and the effects of reactants preheating on the decease of the duration of this delay is observed. Also, the nonequilibrium coupling between the scalar dissipation rate and the flame structure is revealed as quenching frequently appears within the braids. To describe this phenomenon more accurately, work is underway to construct a higher order scheme which can provide better resolution at the regions of strong strain rates.

\section{ACKNOWLEDGMENT}

The authors appreciate the support of NASA Lewis Research Center in providing computer time on the CRAY-XMP. The help of Mr. Don Lovell of Flow Research Company in vectorizing the codes and in providing graphics routines is gratefully acknowledged.

\section{REFERENCES}

1. Bilger, R.W.: Turbulent Flows with Nonpremixed Reactants. Turbulent Reacting Flows, R.W. Bilger, P.A. Libby, and F.A. Williams, eds., SpringerVerlag, Berlin, 1980, pp. 65-113.

2. Donaldson, C. duP.; and Varma, A.K.: Remarks on the Construction of a Second-Order Closure Description of Turbulent Reacting Flows. Combust. Sci. and Technol., vo1. 13, nos. 1-6, 1976, pp. 55-78. 
3. Givi, P.; Ramos, J.I.; and Sirignano, W.A.: Turbulent Reacting Concentric Jets - Comparison Between PDF and Moment Calculations. Dynamics of Flames and Reactive Systems, J.R. Bowen, et al., eds., AIAA, 1984, pp. 384-420.

4. Bilger, R.W.: Turbulent Jet Diffusion Flames. Prog. Energy Combust. Sci.. vol. 1, 1976, pp. 87-109.

5. Lockwood, F.C.; and Naguib, A.S.: The Prediction of the Fluctuations in the Properties of Free, Round-Jet, Turbulent, Diffusion Flames. Combust. Flame, vol. 24, 1975, pp. 109-124.

6. Givi, P.; Sirignano, W.A.; and Pope, S.B.: Probability Calculations for Turbulent Jet Flows with Mixing and Reaction of $\mathrm{NO}$ and $\mathrm{O}_{3}$. Combust. Sci. Technol., vol. 37, nos. 1-2, 1984, pp. 59-78.

7. Nguyen, T.V.; and Pope, S.B.: Monte Carlo Calculations of Turbulent Diffusion flames. Combust. Sci. Technol., vol. 42, nos. 1-2, 1984, pp. 13-45.

8. Pope, S.B.; and Correa, S.M.: Joint PDF Calculations of a Non-Equilibrium Diffusion Flame. Twenty-First Symposium (International) on Combustion, Combustion Institute, Pittsburgh, PA, to appear, 1987.

9. Ghoniem, A.F.: Computational Methods in Turbulent Reacting Flows. Reacting Flows: Combustion and Chemical Reactors, (Lectures in Applied Mathematics, Vol. 24), G. Ludford, ed., American Mathematical Society, 1986, pp. 199-265.

10. Oran; E.A.; and Boris, J.P.: Numerical Simulation of Reactive Flows, to be published by Elsevier Science Publishing Co., 1987.

11. Corcos, G.M.; and Sherman, F.S.: The Mixing Layer; Deterministic Mode1s of a Turbulent Flow. Part 1: Introduction and the Two-Dimensional Flow. J. Fluid Mech., vol. 139, Feb. 1984, pp. 29-65.

12. Grinstein, F.F.; Oran, E.S.; and Boris, J.P.: Numerical Simulations of Symmetric Mixing in Planar Shear Flows. NRL-MR-5621, Naval Research Lab, 1985. (Avail. NTIS, AD-A158024).

13. Riley, J.J.; Metcalfe, R.W.; and Orszag, S.A.: Direct Numerical Simulations of Chemically Reacting Turbulent Mixing Layers. Phys. Fluids, vol. 29, no. 2, Feb. 1986, pp. 406-422.

14. McMurtry, P.A., et al.,: Direct Numerical Simulations of a Reacting Mixing Layer with Chemical Heat Release. AIAA J., vol. 24, no. 6, June 1986, pp. $962-970$.

15. Givi, P.; Jou, W.H.; and Metcalfe, R.W.: Flame Extinction in a Temporarily Evolving Mixing Layer. Twenty-First Symposium (International) on Combustion, The Combustion Institute, Pittsburgh, PA, to appear, 1987.

16. Givi, P.; and Jou, W.H.: Mixing and Chemical Reaction in a Spatially Developing Mixing Layer. Presented at the Central States Section Meeting of The Combustion Institute, Cleveland, $\mathrm{OH}$, May 1986. 
17. Ghoniem, A.F.; Chorin, A.J.; and Oppenheim, A.K.: Numerical Modelling of Turbulent Flow in a Combustion Tunnel. Phil. Trans. R. Soc. London $A$, Vol. 304, no. 1484, Mar. 9, 1982, pp. 303-325.

18. Sethian, J.A.: Turbulent Combustion in Open and Closed Vessels. J. Comput. Phys., vol. 54, no. 3, June 1984, pp. 425-456.

19. Ashurst, W.T.; and Barr, P.K.: Lagrangian-Eulerian Calculation of Turbulent Diffusion Flame Propagation. SAND80-9950, Sandia National Laboratories, 1982.

20. Lin, P.; and Pratt, D.T.: Numerical Simulation of a Plane Turbulent Mixing Layer, With Applications to Isotherma1, Rapid Reactions. AIAA Paper 87-0224, Jan. 1987.

21. Roshko, A.; and Roberts, F.A.: Effects of Periodic Forcing on Mixing in Turbulent Shear Layers and Wakes. AIAA Paper 85-0570, Jan. 1985.

22. Ghoniem, A.F.; and Ng, K.K.: Numerical Study of the Dynamics of a Forced Shear Layer. Phys. Fluids, vol. 30, no. 3, Mar. 1987, pp. 706-721.

23. Chorin, A.M.: Numerical Study of Slightly viscous Flow. J. Fluid Mech., vol. 57, pt. 4, Mar. 6, 1973, pp. 785-796.

24. Hald, 0.H.: The Convergence of Vortex Methods for Eulers Equations. II. SIAM J. Numer. Ana 1., vol. 16, no. 5, Oct. 1979, pp. 726-755.

25. Beale, J.T.; and Majda, A.: Vortex Methods. II. Higher Order Accuracy in Two and Three Dimensions. Math. Comput., vol. 39, no. 159, July 1982, pp. 29-52.

26. Ghoniem, A.F.; and Cagnon, Y.: Vortex Simulation of Laminar Recirculating Flow. J. Comput. Phys., vol. 68, no. 2, Feb. 1987, pp. 346-377.

27. Ghoniem, A.F.; and Oppenheim, A.K.: Numerical Solution for the Problem of Flame Propagation by the Random Element Method. AIAA J., vol 22, no. 10, Oct. 1984, pp. 1429-1435.

28. Ghoniem, A.F.; and Sherman, F.S.: Grid-Free Simulation of Diffusion Using Random Walk Methods. J. Comput. Phys., vol. 61, Nov. 1985, pp. 1-37.

29. Koochesfahani, M.M.: Experiments on Turbulent Mixing and Chemical Reactions in a Liquid Mixing Layer. Ph.D. Thesis, California Institute of Technology, 1984.

30. Masutani, S.M.; and Bowman, C.T.: The Structure of a Chemically Reacting Plane Mixing Layer. J. Fluid Mech., vol. 172, Nov. 1986, pp. 93-126.

31. Givi, P.; Ramos, J.I.; and Sirignano, W.A.: Probability Density Function Calculations in Turbulent Chemically Reacting Round Jets, Mixing Layers and One-Dimensional Reactors. J. Non-Equilib. Thermodyn., vol. 10, no. 2, 1985, pp. 75-104. 
32. Batt, R.G.: Turbulent Mixing of Passive and Chemically Reacting Species in a Low-Speed Shear Layer. J. Fluid Mech., vol. 82, pt. 1, Aug. 19, 197\%, pp. 53-95.

33. Broadwell, J.E.; and Breidenthal, R.E.: A Simple Model of Mixing and Chemical Reaction in a Turbulent Shear Layer. J. Fluid Mech., vol. 125, Dec. 1982, pp. 397-410.

34. Ghoniem, A.F.; Heidarienejad, G.; and Krishnan, A.: Computation of a Thermally Stratified Mixing Layer. J. Comput. Phys., submitted for publication, 1987.

35. Oster, D.; and Wygnanski, I.: The Forced Mixing Layer Between Parallel Streams. J. Fluid Mech., vol. 123, Oct. 1982, pp. 97-130.

36. Browand, F.K.; and Ho, C.H.: The Mixing Layer: An Example of Quasi TwoDimensional Turbulence. J. Mec. Theor. Appl., Special Supplement, 1983, pp. $99-120$.

37. Tsuji, H.: Counterflow Diffusion Flames. Prog. Energy Combust. Sci., vol. 8, 1982, pp. 93-119.

38. Liew, S.K.; Moss, J.B.; and Bray, K.N.C.: Predicted Structure of Stretched and Unstretched Methane-Air Diffusion Flame. Dynamics of Flames and Reactive Systems, J.R. Bowen, et al., eds., AIAA, 1984, pp. 305-319.

39. Peters, N.: Laminar Diffusion Flamelet Models in Non-Premixed Turbulent Combustion. Prog. Energy Combust. Sci., vol. 10, 1984, pp. 319-339.

TABLE I. -

\begin{tabular}{|c|c|c|}
\hline \multicolumn{3}{|c|}{ Frequency locked region } \\
\hline$\Omega$ & Calculated & Measured (ref. 36) \\
\hline 0.5 & $3 \leqq x \leqq 4$ & $2.66 \leqq x \leqq 5.33$ \\
1.0 & $2 \leqq x \leqq 3$ & $1.33 \leqq x \leqq 2.66$ \\
\hline
\end{tabular}

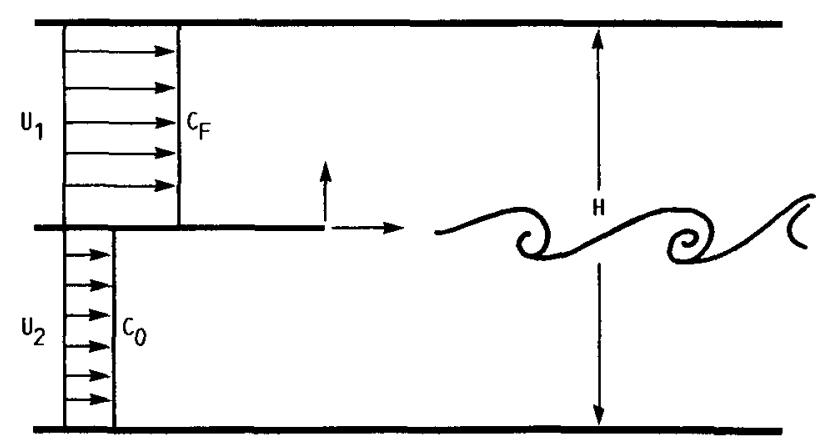

FIGURE 1. - SCHEMATIC DIAGRAM OF THE SHEAR LAYER MODEL. 


\section{ORIGINAL PAGE TS \\ OF POOR QUALITY.}

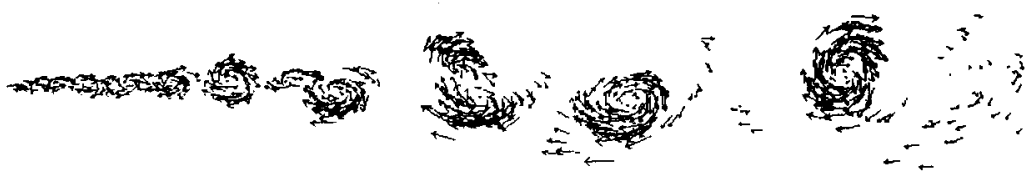

$\mathrm{U} 2=0.3333, \mathrm{RE}=24000 ; \mathrm{TIME}=28.00$

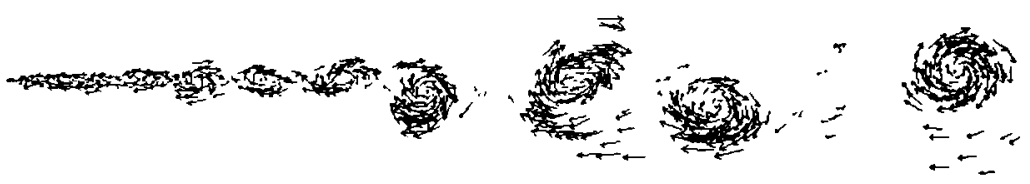

$U 2=0.3333, \operatorname{RE}=24000 ;$ TIME $=29.00$

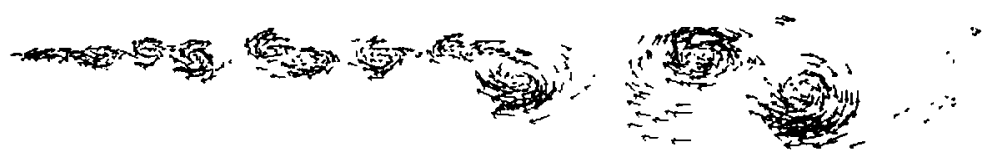

U2 $=0.3333, R E=24000: T I M E=30,00$

FIGURE 2. - VORTICITY FIELD AT RE $=24000$, U2/U1 $=1 / 3$.

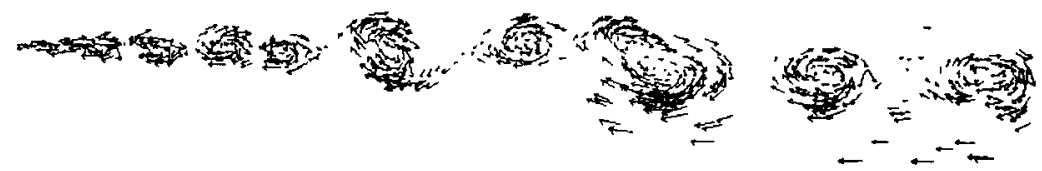

$U 2=0.3333, \operatorname{RE}=10000 ;$ TIME $=28.00$

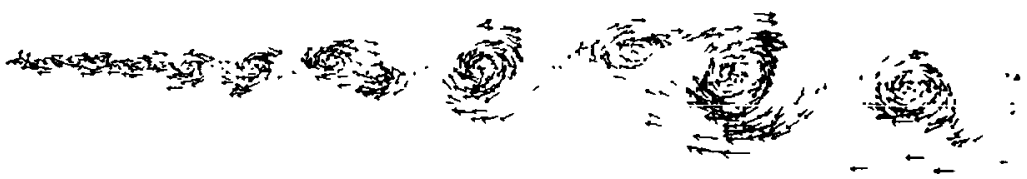

$\mathrm{U} 2=0.3333, \mathrm{RE}=10000 ;$ TIME $=29.00$

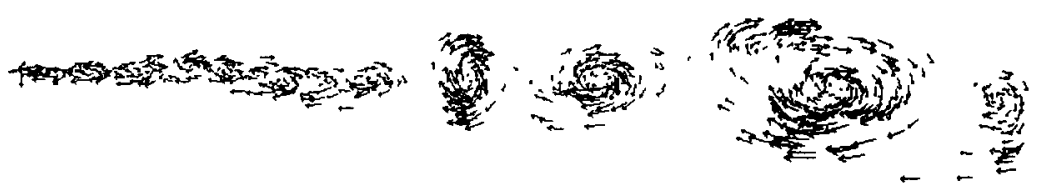

$\mathrm{U} 2=0.3333, \mathrm{RE}=10000 ; \mathrm{TIME}=30.00$

FIGURE 3. - VORTICITY FIELD AT RE $=10000$, U2/U1 $=1 / 3$. 


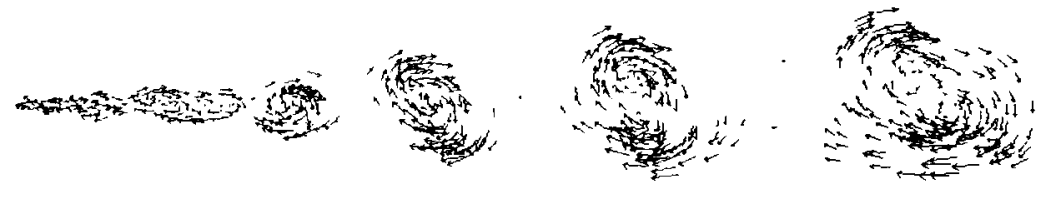

U2 $=0.3333, \operatorname{RE}=4000 ;$ TIME $=28.00$

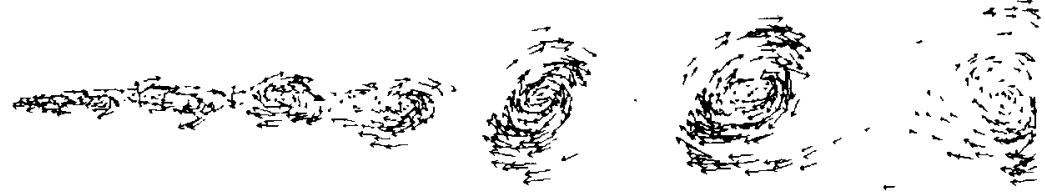

$\mathrm{U} 2=0.333 \overline{5}, \mathrm{RE}=4000: \mathrm{TIME}=29.00$

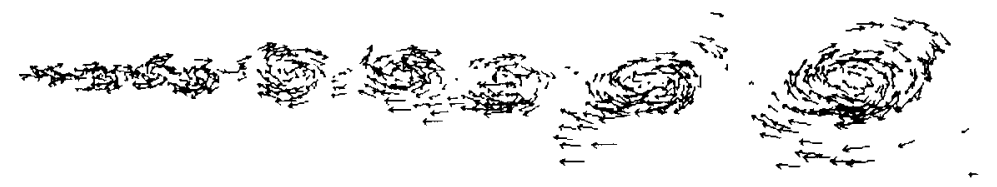

$\mathrm{U} 2=0.3333, \mathrm{RE}=4000 ; \mathrm{TIME}=30.00$

FIGURE 4. - VORTICITY FIELD AT RE $=4000, \mathrm{U} 2 / \mathrm{U} 1=1 / 3$.

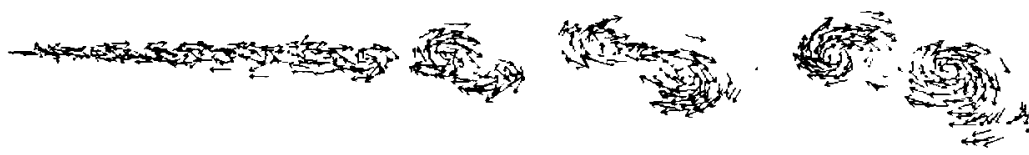

$\mathrm{U} 2=0.5000, \mathrm{RE}=10000: \operatorname{TIME}=28.00$

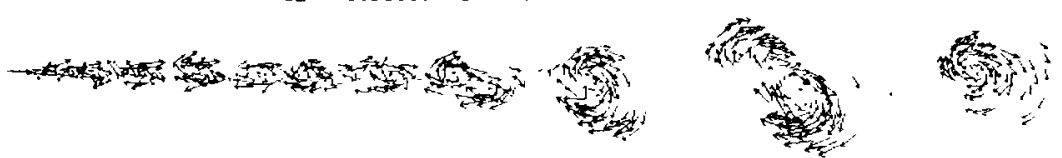

$\mathrm{U} 2=0.5000, \mathrm{RE}=10000 ; \mathrm{TIME}=29.00$

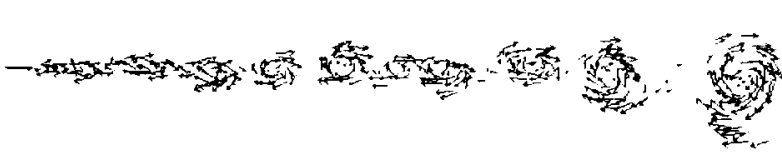

U2 $=0.5000, R E=10000 ;$ TIME $=30.00$

FIGURE 5, - VORTICITY FIELD AT RE $=10000, \mathrm{U} 2 / \mathrm{U} 1=0.5$. 


\section{ORIGINAL PAGE IS \\ OF POOR QUALITY}

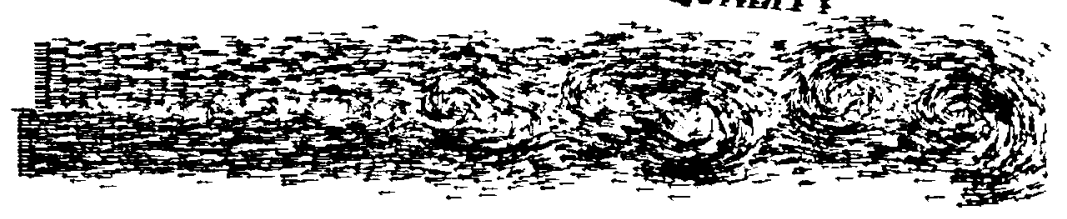

$U 2=0.5000, \operatorname{RE}=10000:$ TIME $=28.00$

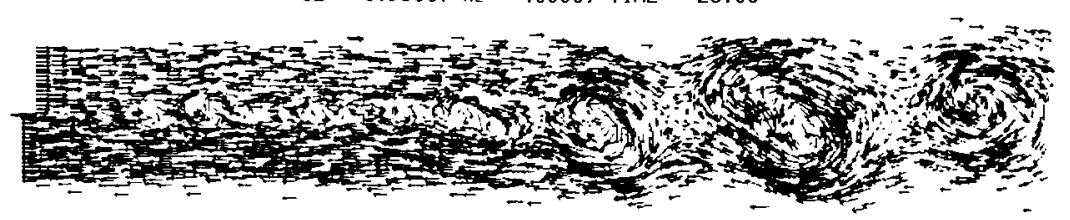

$\mathrm{U} 2=0.5000, \mathrm{RE}_{\mathrm{E}}=10000:$ TIME $=29.00$

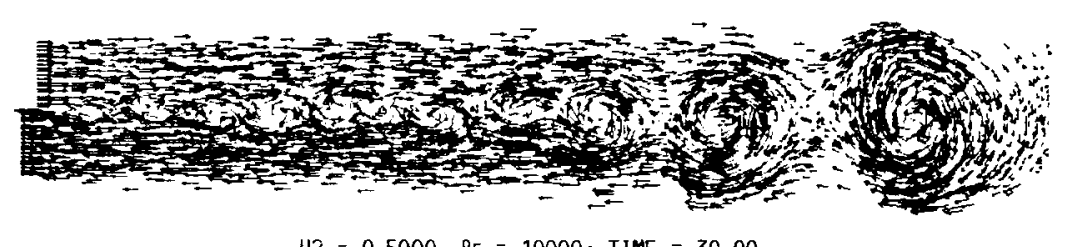

$\mathrm{U} 2=0.5000 \cdot \operatorname{RE}=10000: \operatorname{TIME}=30.00$

FIGURE 6. - SCALAR'S VELOCITY FIELD AT RE $=10000, \mathrm{U} 2 / \mathrm{U1}=0 . \mathrm{b}$.

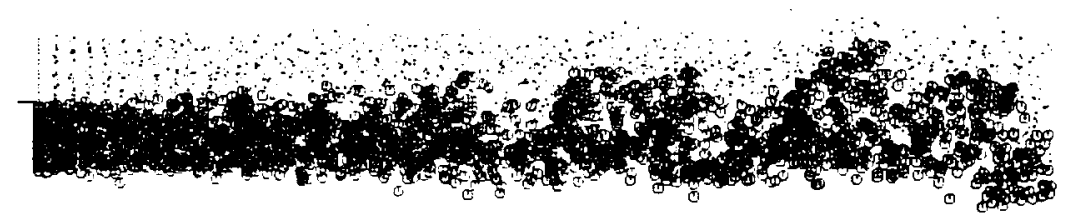

$\mathrm{U} 2=0.5000 \cdot \mathrm{RE}=10000:$ TIME $=28.00$

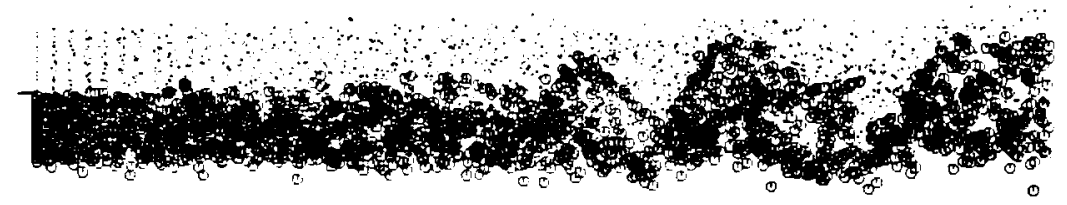

$\mathrm{U} 2=0.5000, \mathrm{RE}=10000 ;$ TIME $=29.00$

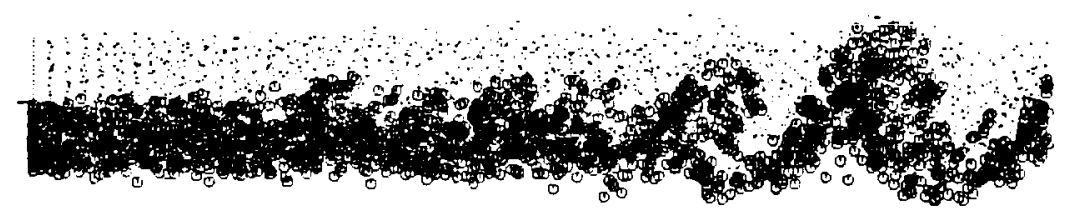

$\mathrm{U} 2=0.5000, \mathrm{RE}=10000:$ TIME $=30.00$

FiguRE 7. - CONCENTRATION FIELD AT RE $=10000, \mathrm{PE}=4000, \mathrm{U} 2 / \mathrm{U1}=0.5$. 


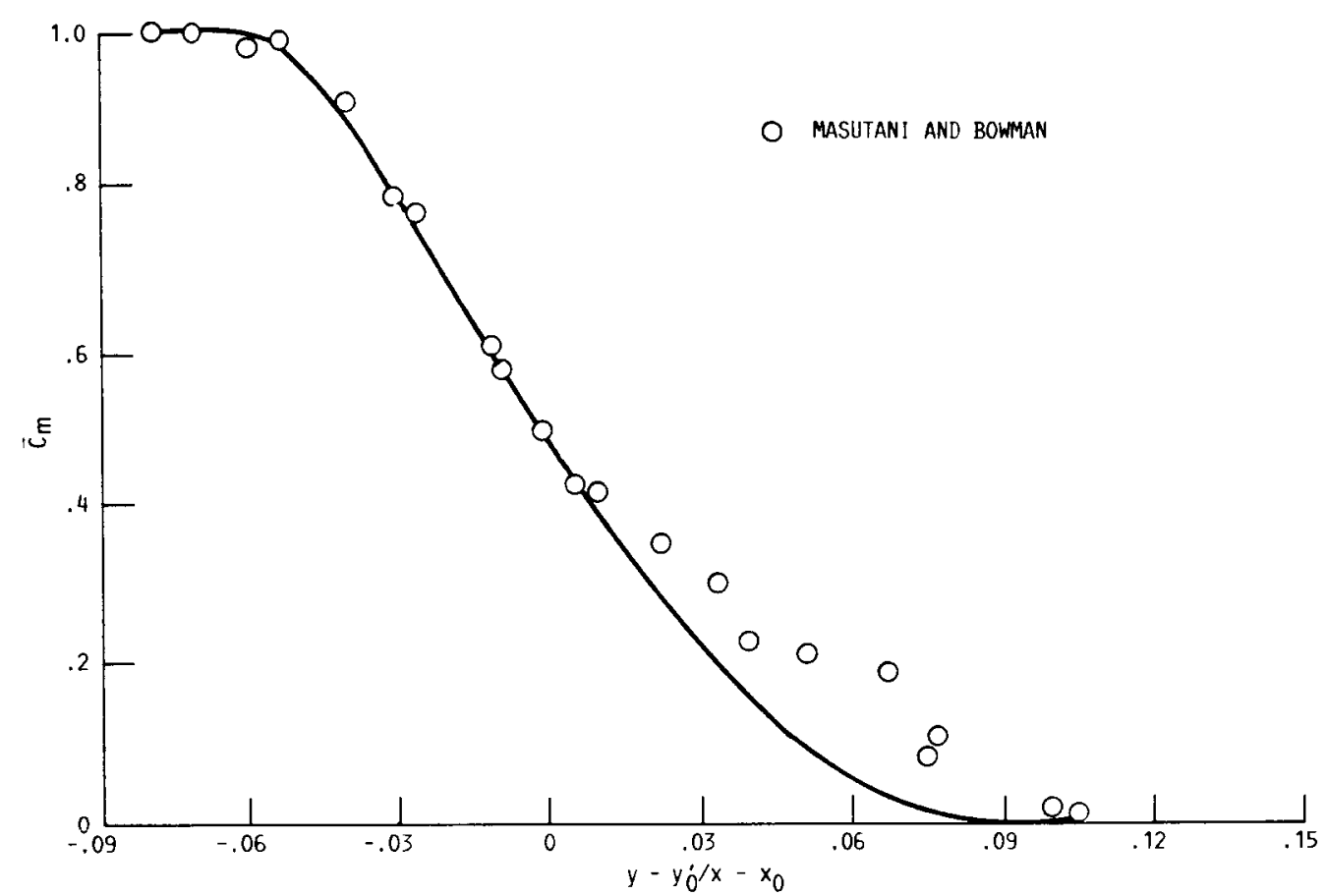

FigURE 8. - NORMALIZED MEAN CONCENTRATION PROFILE AS A FUNCTION OF THE CROSS-STREAM COORDINATE.

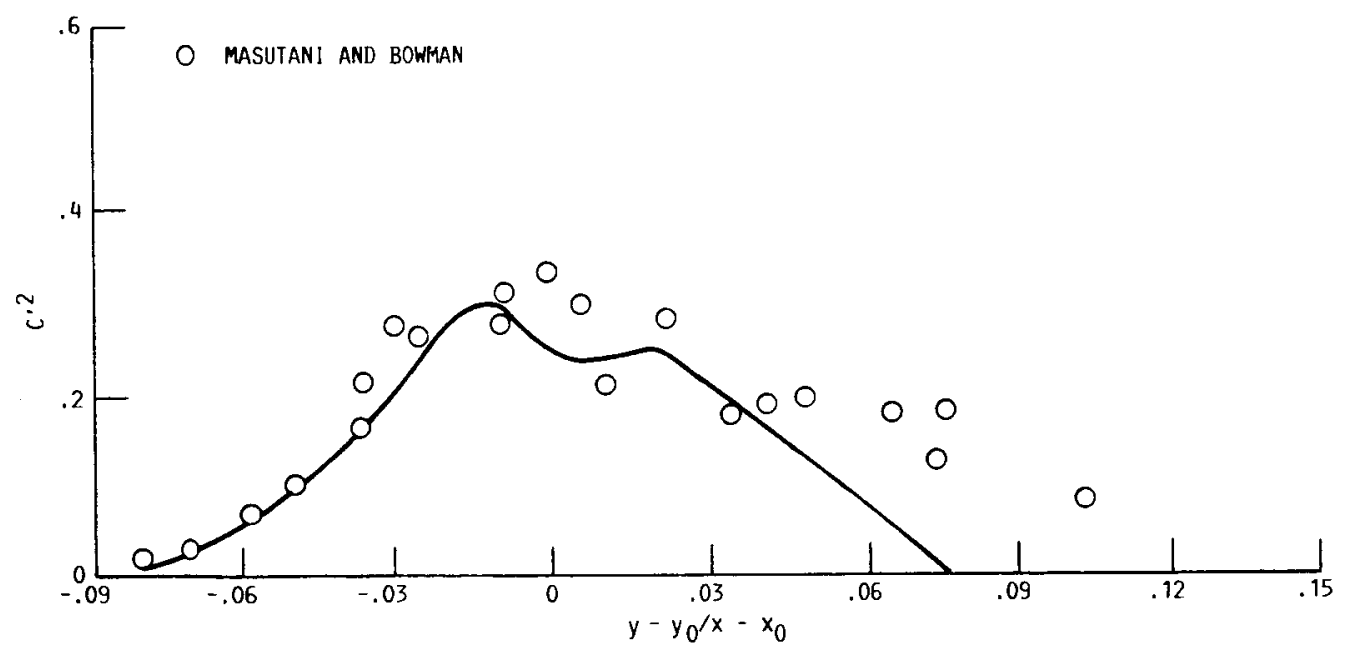

FIGURE 9. - NORMALIZED RMS CONCENTRATION PROFILE AS A FUNCTION OF THE CROSS-STREAM COORDINATE. 


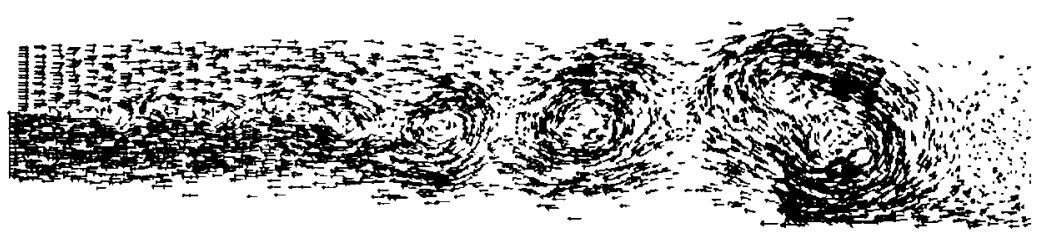

$\mathrm{U} 2=0.3333, \mathrm{RE}=10000:$ IIME $=19.00$

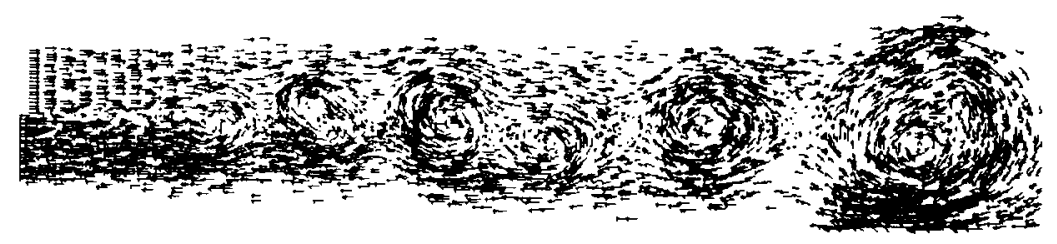

$\mathrm{U} 2=0.3333, \mathrm{RE}=10000 ; \mathrm{TIME}=20.00$

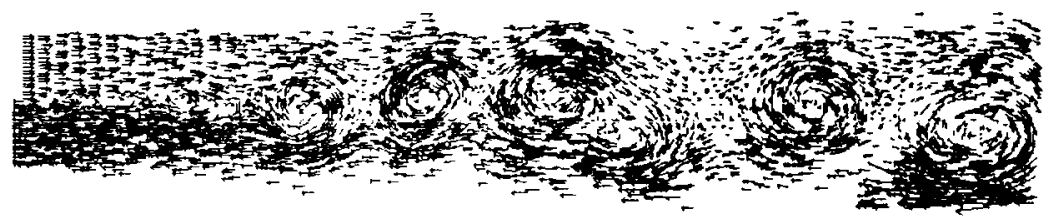

$\mathrm{U} 2=0.3333, \mathrm{RE}=10000 ; \mathrm{TIME}=21.00$

FIGURE 10. - SCALAR'S VELOCITY FIELD AT RE $=10000, U 2 / \mathrm{U} 1=1 / 3$.

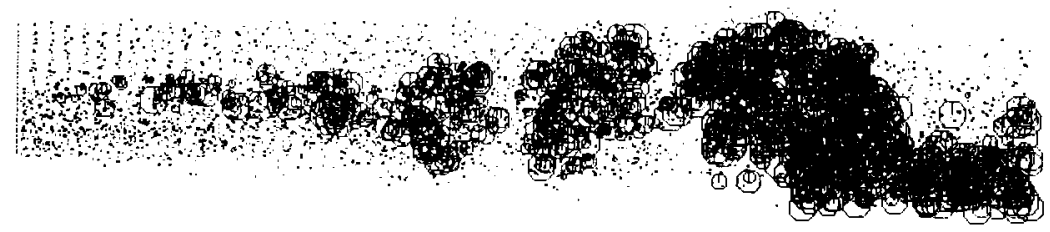

$U 2=0.3333, R E=10000 ;$ TIME $=19.00$

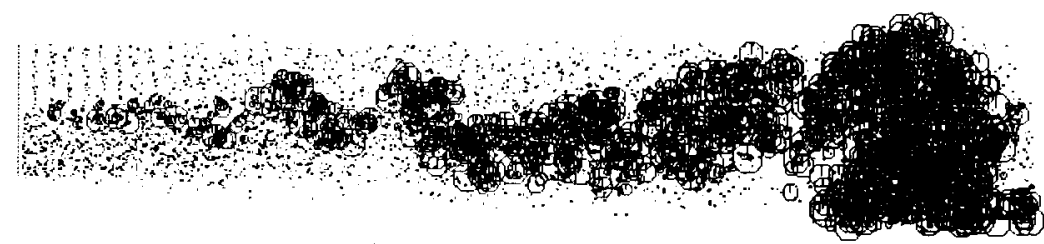

$\mathrm{U} 2=0.3333, \mathrm{RE}=10000: \mathrm{TIME}=20.00$

FIGURE 11. - PRODUCT CONCENTRATION FIELD, RE $=10000, P E=4000, U 2 / U 1=1 / 3$, ISOTHERMAL REACTING LAYER. 


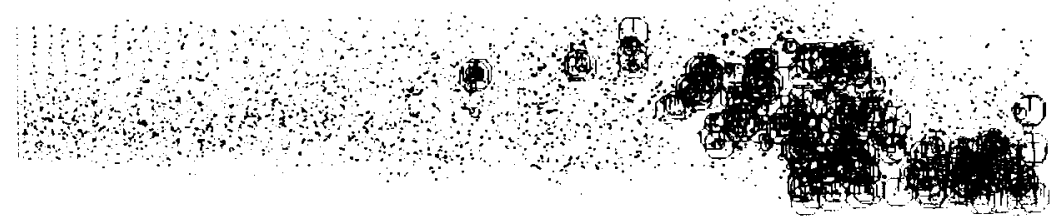

$\mathrm{U} 2=0.3333, \mathrm{RE}=10000 ; \mathrm{TIME}=19.00$

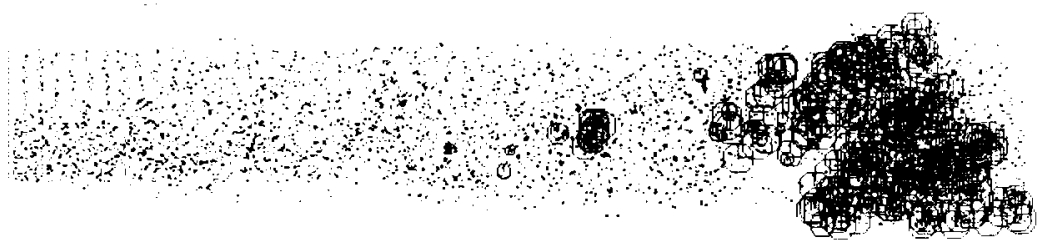

$\mathrm{U} 2=0.3333, \mathrm{RE}=10000 ; \operatorname{TIME}=20.00$

FIGURE 12. - PRODUCT CONCENTRATION FIELD, VARIABLE TEMPERATURE REACTING LAYER.

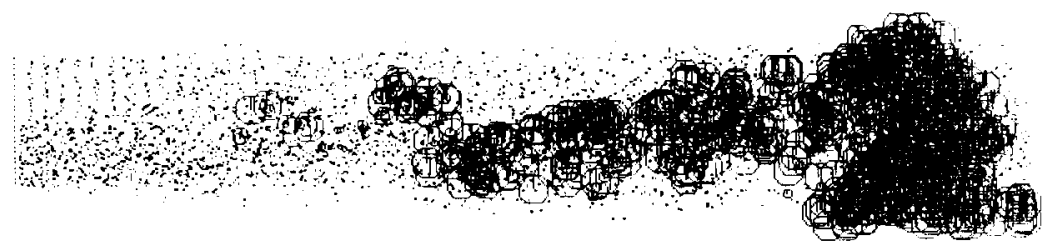

$U 2=0.3333, \operatorname{RE}=10000 ; T I M E=20.00$

FIGURE 13. - PRODUCT CONCENTRATION FIELD, VARIABLE TEMPERATURE REACTING LAYER.

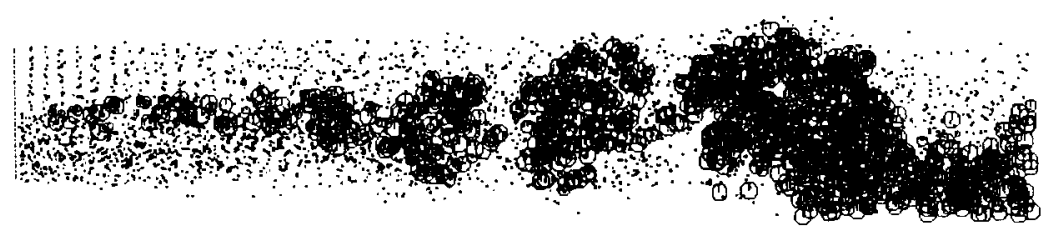

U2 $=0.3333 . \operatorname{RE}=10000 ;$ TIME $=19.00$

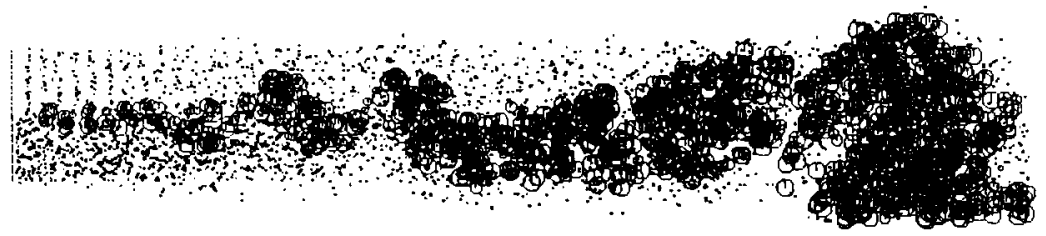

$\mathrm{U} 2=0.3333, \mathrm{RE}=10000 ;$ TIME $=20.00$

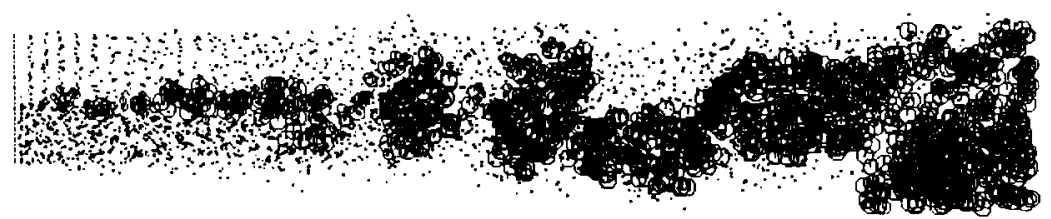

$\mathrm{U} 2=0.3333, \mathrm{RE}=10000 ; \mathrm{TIME}=21.00$

FIGURE 14. - PRODUCT CONCENTRATION FIELD, VARIABLE TEMPERATURE REACTING LAYER. 


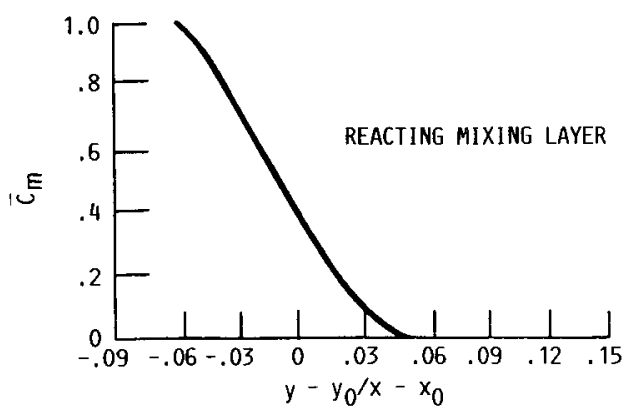

FIGURE 15. - NORMALIZED MEAN CONCENTRATION PROFILE AS A FUNCTION OF THE CROSSSTREAM COORDINATE.

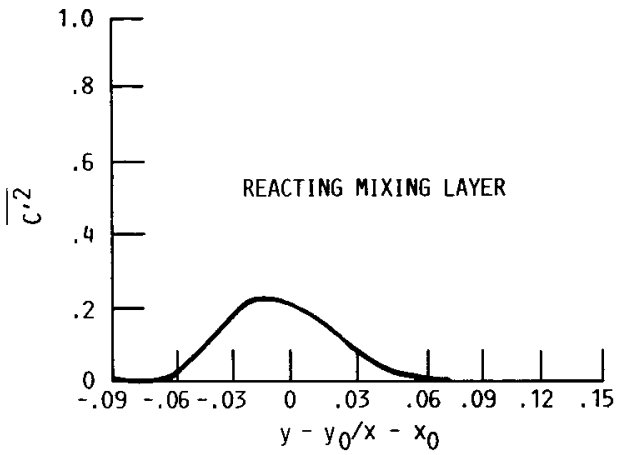

FIGURE 16. - NORMALIZED RMS CONCENTRATION PROFILE AS A FUNCTION OF THE CROSSSTREAM COORDINATE.

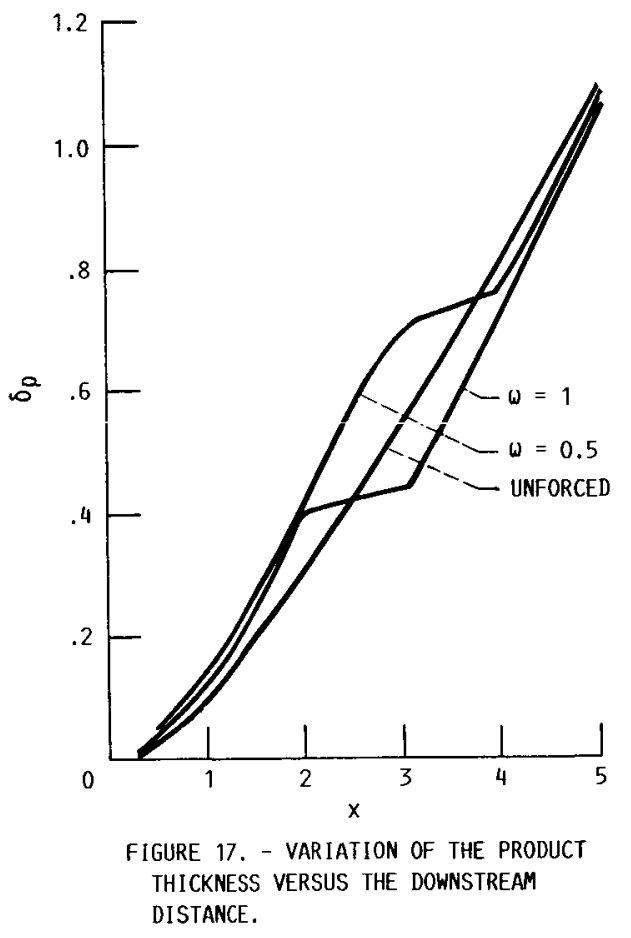




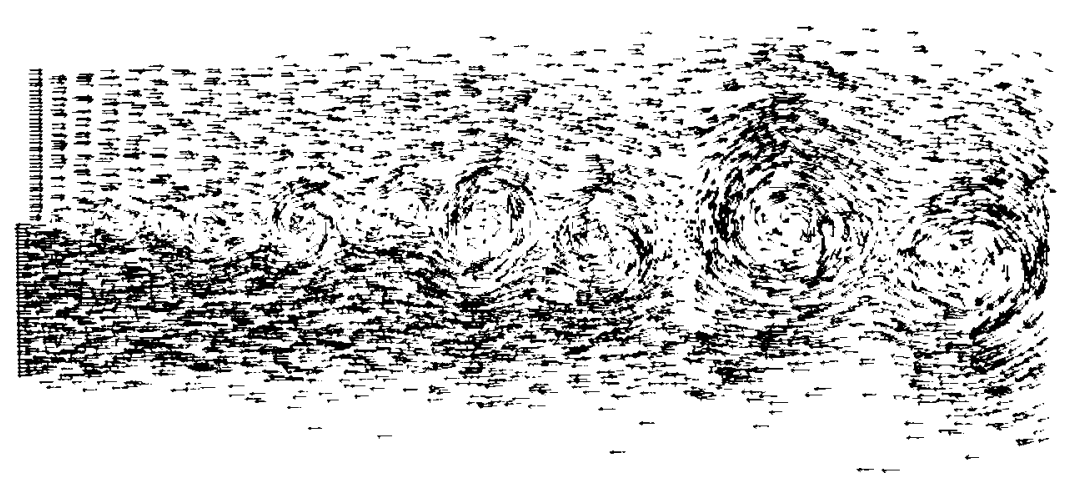

U2 $=0.3333$, RE $=10000:$ TIME $=19.00$

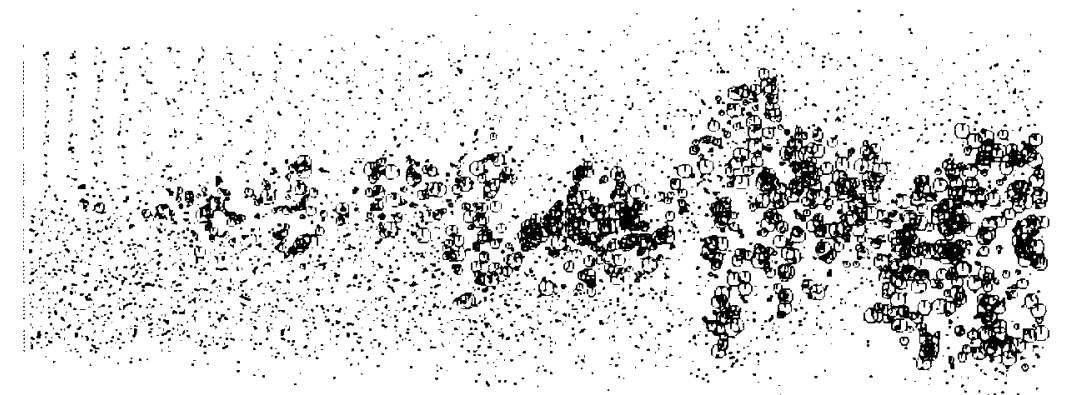

$\mathrm{U} 2=0.3333, \mathrm{RE}=10000: \mathrm{TIME}=19.00$

FIGURE 18. - TEMPERATURE FIELD FOR REACTING MIXING LAYER.

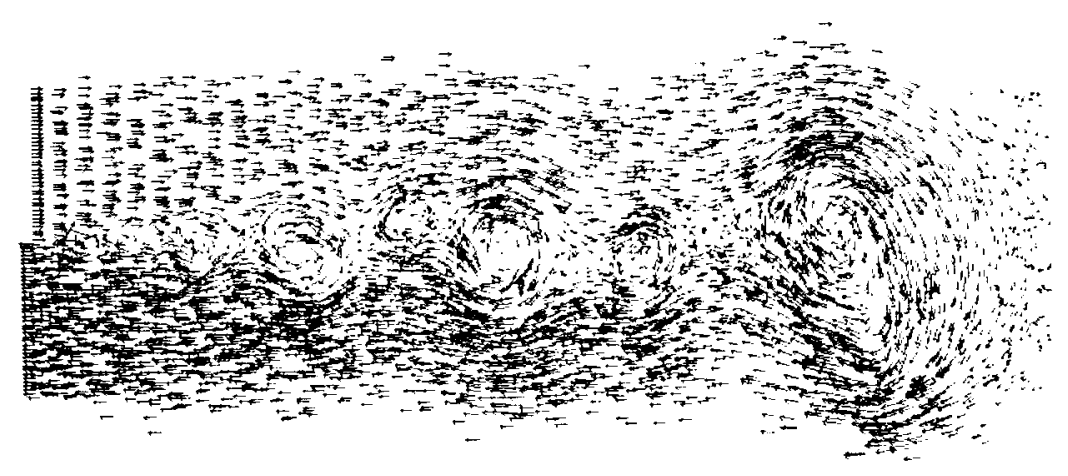

$U 2=0.3333, \operatorname{RE}=10000: T I M E=21.00$

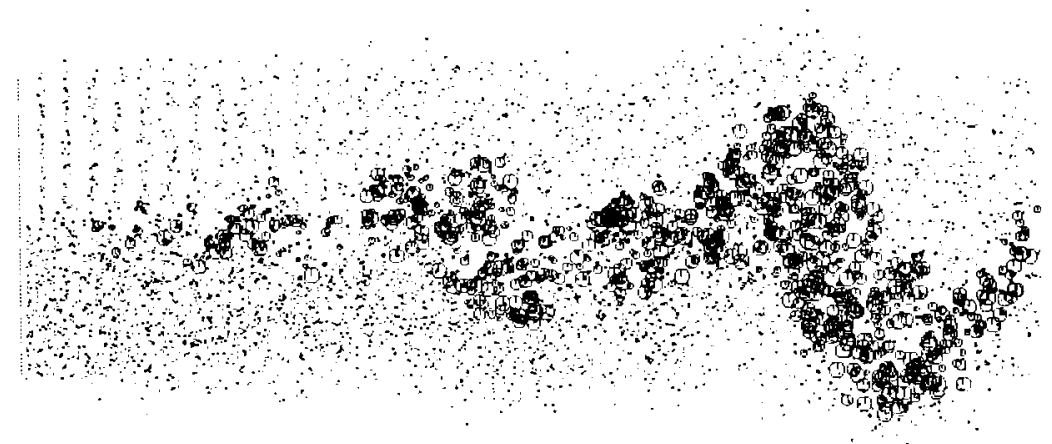

$\mathrm{U} 2=0.3333 . \operatorname{RE}=10000 ; \mathrm{TIME}=21.00$

FIGURE 19. - TEMPERATURE FIELD FOR REACTING MIXING LAYER. 


\begin{tabular}{|c|c|c|c|c|}
\hline$\prod_{\substack{\text { National Aeronautics and } \\
\text { Space Adrministration }}}$ & eport Docu & ntation & & \\
\hline $\begin{array}{l}\text { 1. Report No. } \\
\text { NASA TM-100133 } \\
\text { ICOMP-87-4 }\end{array}$ & 2. Government Ac & n No. & 3. Recipient's Cata & \\
\hline 4. Title and Subtitle & 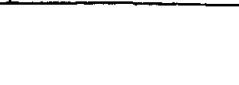 & & $\begin{array}{l}\text { 5. Report Date } \\
\text { August } 19\end{array}$ & \\
\hline $\begin{array}{l}\text { Vortex-Scalar Element } c \\
\text { Flame Stabilized on a } P\end{array}$ & $\begin{array}{l}\text { lations of } \\
\text { Mixing Laye }\end{array}$ & ffusion & $\begin{array}{r}\text { 6. Performing Orga } \\
505-62-21\end{array}$ & on Code \\
\hline $\begin{array}{l}\text { 7. Author(s) } \\
\text { Ahmed F. Ghoniem and Pe }\end{array}$ & Givi & & $\begin{array}{l}\text { 8. Performing Orga } \\
\text { E-3683 }\end{array}$ & on Report No. \\
\hline & & & 10. Work Unit No. & \\
\hline 9. Performing Organization Name and Addre & & & & \\
\hline $\begin{array}{l}\text { National Aeronautics an } \\
\text { Lewis Research Center } \\
\text { Cleveland Ohio } 44735\end{array}$ & ace Administ & ion & 11. Contract or Grar & \\
\hline 12. Sponsoring Agency Name and Address & & & $\begin{array}{l}\text { 13. Type of Report } \\
\text { Technica }\end{array}$ & morandum \\
\hline $\begin{array}{l}\text { National Aeronautics an } \\
\text { Washington, D.C. } 20546\end{array}$ & ace Adminis & ion & 14. Sponsoring Ager & \\
\hline $\begin{array}{l}\text { 15. Supplementary Notes } \\
\text { Amed F. Ghoniem, Dept. of Me } \\
\text { Massachusetts 02 } 139 \text { (Work su } \\
\text { the National Science Foundat } \\
\text { chusetts Inst itute of Techno } \\
\text { NASA Lewis Research Center ( } \\
\text { Research Company, Kent, Wash }\end{array}$ & $\begin{array}{l}\text { cal Engineering } \\
\text { ed by the Air F } \\
\text { rant CPE-840481 } \\
\text { Peyman Givi, } \\
\text { funded under Sp } \\
\text { n. }\end{array}$ & $\begin{array}{l}\text { ssachuset } \\
\text { Office o } \\
\text { d the Edg } \\
\text { i tute for } \\
\text { Act Agreer }\end{array}$ & $\begin{array}{l}\text { ittute of Techno } \\
\text { tific Research } \\
\text { ssociate Profes } \\
\text { ational Mechani } \\
\text { 9066G); on leav }\end{array}$ & $\begin{array}{l}\text { Cambridge } \\
\text { AFOSR 84-0356 } \\
\text { hip at Massa- } \\
\text { n Propulsion, } \\
\text { om Flow }\end{array}$ \\
\hline $\begin{array}{l}\text { 16. Abstract } \\
\text { The vortex-scalar eleme } \\
\text { cretize the region of } \\
\text { temperature fields, is } \\
\text { reacting mixing layer. } \\
\text { Reynolds and Peclet num } \\
\text { reacting flow, the mean } \\
\text { agreement with experime } \\
\text { that for temperature-in } \\
\text { ately downstream of the } \\
\text { reacting flow with Arrh } \\
\text { the reactants temperatc } \\
\text { forcing changes the str } \\
\text { ing and reaction, in ac } \\
\text { the braids in the nonec } \\
\text { to the temperature drop }\end{array}$ & $\begin{array}{l}\text { ethod, a scl } \\
\text { vorticity al } \\
\text { ized in the } \\
\text { putations al } \\
\text { without res } \\
\text { fiuctuatiol } \\
\text { measuremen } \\
\text { indent kinet } \\
\text { itter plate } \\
\text { is kinetics } \\
\text { before sign } \\
\text { lre of the } \\
\text { lance with } \\
\text { brium kinet } \\
\text { ociated witl }\end{array}$ & $\begin{array}{l}\text { which } \\
\text { calar e } \\
\text { nerical } \\
\text { erforme } \\
\text { :ing to } \\
\text { ofiles } \\
\text { Result } \\
\text { the ch } \\
\text { re mixi } \\
\text { an ign } \\
\text { ant che } \\
\text { and } \\
\text { imental } \\
\text { case ca } \\
\text { ie large }\end{array}$ & $\begin{array}{l}\text { es vortex e } \\
\text { s to represe } \\
\text { tions of a } \\
\text { a diffusion } \\
\text { ence models } \\
\text { onserved sca } \\
\text { the reactins } \\
\text { reaction be } \\
\text { irts. Resul } \\
\text { delay, which } \\
\text { reaction oce } \\
\text { tantly the } \\
\text { its. Strong } \\
\text { local flame } \\
\text { ctive fluxe }\end{array}$ & $\begin{array}{l}\text { nts to dis- } \\
\text { species or } \\
\text { dimensional } \\
\text { me at high } \\
\text { n the non- } \\
\text { show good } \\
\text { ow indicate } \\
\text { is immedi- } \\
\text { or the } \\
\text { pends on } \\
\text { Harmonic } \\
\text { s of mix- } \\
\text { etch within } \\
\text { iching due }\end{array}$ \\
\hline $\begin{array}{l}\text { 17. Key Words (Suggested by Author(s)) } \\
\text { Vortex methods; React ir } \\
\text { layers; Turbulence chen } \\
\text { Interactions }\end{array}$ & $\begin{array}{l}\text { ows; Mixing } \\
y \text {; }\end{array}$ & $\begin{array}{l}\text { 18. Distributi } \\
\text { Uncla } \\
\text { STAR }\end{array}$ & on - unlimit & \\
\hline $\begin{array}{l}\text { 19. Security Classif. (of this report) } \\
\text { Unc lass if ied }\end{array}$ & $\begin{array}{r}\text {. Security Classif. (of } \\
\text { UnC }\end{array}$ & ified & \begin{tabular}{|c} 
21. No of pages \\
25
\end{tabular} & $\begin{array}{r}\text { 22. Price* } \\
\text { A02 }\end{array}$ \\
\hline
\end{tabular}

